\section{Clinical Hotes:}

\section{MEDICAL, SURGICAL, OBSTETRICAL, AND THERAPEUTICAL.}

\section{POISONING BY ACONITE AND BELLADONNA.} By Hugh R. Jones, M.A., M.B. Cantad.

Ax old lady, seventy-five years of age, swallowed by mistake a large tablespoonful of a mixture of equal parts of aconite and belladonna liniments. She vomited almost immediately, and an emetic of salt-and-water was administered by her friends. On the arrival of myself and my friend Dr. Hunt Cooke at 10 P.M. (three-quarters of an hour after the liniment had been swallowed), she was retching and romiting, and complaining of a curious sensation in her throat and of tightness across her chest, which prevented her lying down. Pulse 98, slightly irregular, small and weak; pupils equal, not dilated. I washed her stomach out with warm water and gave her two drachms of brandy at once, and afterwards the white of an egg beaten up with two drachms of brandy and half an ounce of water every hour, in small quantities at a time. This appeared to relieve the vomiting, which was almost incessant. The pulse grew weaker and weaker, slower and more irregular, until an attack of faintness canıe on at 10.30 P.M. The pulse was then 64 , and imperceptible at the wrist; when felt at the elbow it was weak, extremely irregular, and intermittent. The extremities were cold. Respiration ceased, and the patient became quite rigid, the jaws being firmly closed, with conjugate deviation of the eyes to the left. This attack was relieved by ten minims of brandy given hypodermically. The pulse improved slightly, but gradually failed again, until at 10.50 P.M. she had a second attack, similar in all respects, which was relieved in the same way. From this time there was a gradual though tardy improvement in the pulse and marked diminution of retching, so that at 1 A.M. I was able to leave her fairly comfortable. During the greater part of the time she was slightly delirious. The following day she had great abdominal soreness and occipital headache, but had otherwise quite recovered. The symptoms seem to have been almost entirely due to aconite, the delirium alone being possibly assignable to belladonna. The effect of the brandy was so marked each time it was given that I considered it unnecessary to use digitalis or any other drug.

Llanwddyn.

\section{A PLEA FOR THE USE OF THE RHINOSCOPE.}

By WM. Robertson, M.D.,

SURGEON TO THE THROAT AND EAR HOSPITAL, NEWCASTLE-ON-TYNE.

THE following cases, although not of exceptional interest, supply sufficient proof of the advisability, as a matter of routine, of the use of the post-nasal mirror in every case of the description referred to.

The first case is that of Mr. R_- who came to me conplaining of deafness of four years' duration. A watch was heard on contact only, and, with the exception of some cerumen in both meatus, the case further presented very little evidence of disease to the ordinary methods of investigation. The pharynx was healthy, and the nose remarliably free from catarrh or stenosis. The history was that he had suffered from a chancre four years ago, that subsequently he became deaf, and had remained so ever since. On arriving in England, he had consulted various medical men, all of whom failed to make out any cause for the deafness. With the help of the mirror a curious state of the post-nasal space was noticed. It was found occupied by heaped-up masses of hard, dry crusts. On removing these, an explanation of the deafness was accounted for. Extensive ulceration surrounded the right nares posteriorly, exposing the vomer in the neighbourhood. The tissues in the space were extensively infiltrated, and both tubes choked. Chromic acid was freely applied to the disease, whilst a spray was ordered for constant use. The internal treatment consisted of blue pill and iodide of potassium. In two days the hearing had improved, so that a moderate pitch of the voice could be heard.
The second case, that of Miss I-, is an example of a numerous class who present themselves with symptoms, although indefinite, pointing towards implication of the upper air passages. A prominent symptom in this young lady's case was a severe pressing pain over the root of the neck posteriorly, forcing her to lean forward, in which position she got relief. The other symptoms were periodical attacks of somnolence in the daytime; palpitation, frequently awakening her in the night; depression, and irritability of temper. This state of matters had continued more or less for ten or eleven years, during which term various diagnoses were arrived at: incipient heart disease, commencing tuberculosis in one or other lung, hysteria, want of occupation, \&c. Examination of the nares anteriorly betrayed nothing, with the exception of a marked deviation of the septum to the right, and hypertrophy of tissues over both lower turbinated bones. The pharynx was normal and roomy. With the mirror a large polypus, about the size of a nut, was discovered protruding from the right nares posteriorly. On removing this through the mouth, another equally large was brought into riew, and was snared through the nose. All the symptoms mentioned disappeared, and appropriate treatment of the other lesions finished the case.

One cannot be too careful in examining the nose in patients who present themselves complaining of throat symptoms with no apparent lesion there, for, in examining such, small polypi are often discovered in the posterios regions, while anteriorly the nose appears to be unaffected. Newcastle-on-Tyne.

\section{ABSCESS IN THE FEMORAL REGION SIMULATING HERNIA.}

BY H. V. DRew, M.R.C.S., L.R.C.P.L., LATE RESIDENT SURGEON TO THE TIMARU HOSPITAL, NEW ZEALAND.

KATE O. B- , single, aged about thirty, was admitted into the Timaru Hospital suffering from a fluctuating swelling in the right femoral region, evidently an abscess; temperature $104^{\circ}$. About a fortnight before being admitted she was serving dinner, when, after carrying a somewhat heavy dish, she was seized with violent vomiting and acute pain in the region of the umbilicus. She did not notice any swelling, nor had she previously noticed any, and was before this event in perfect health. Her mistress sent for a medical man, who for some reason could not come. She had complete constipation. This occurred on the Tuesday night. She continued in the same condition until the Thursday morning. Domestic medicine had been resorted to, and aperients had been administered, of course without relief. On the Thursday morning the symptoms suddenly abated, and on the Saturday she was seen by a medical man, who apparently made no diagnosis. Some short time after this she complained of a swelling in the femoral region, and she was sent to the hospital without a word concerning the history of her illness. From the above symptoms the diagnosis of unrelieved (surgically) strangulated femoral hernia was made. The patient declined to allow the abscess to be opened, though suffering from severe constitutional disturbance; so hot fomentations were applied and opium given. The next day the shin sloughed, and a quantity of pus, fæees, and a small slough were discharged. Her temperature fell to normal after the cavity had been cleaned and dusted with iodoform. Freces and gas continued to be discharged for about a fortnight, when the wound gradually granulated up and the patient left the hospital well.

From what I could gather the patient seemed to have suffered acutely, and the strange thing about the case, apart from her fortunate recovery, appears to be the long interval that elapsed before the crisis occurred-viz., from the Tuesday night until the Thursday morning. New Zealand.

THE LewishaM TRAGEDY.-The chemist's assistant who supplied eight grains instead of five drops of liq. strych. by which mistake it will be remembered a Lewisham publican recently lost his life, has been fined $£ 5$ and $12 s$. costs for not labelling the bottle in which it was supplied with the name and address of the seller of the poison, and omitting to cause any entry to be made of the sale in a book kept for the purpose. 